Relations industrielles

Industrial Relations

\title{
Reed C. RICHARDSON : Collective Bargaining by Objectives : A Positive Approach. 2nd ed., New Jersey, Prentice-Hall, 1985, 325 pp., ISBN 0-13-140476-8-01
}

\section{Esther Déom}

Volume 41, numéro 1, 1986

URI : https://id.erudit.org/iderudit/050191ar

DOI : https://doi.org/10.7202/050191ar

Aller au sommaire du numéro

Éditeur(s)

Département des relations industrielles de l'Université Laval

ISSN

0034-379X (imprimé)

1703-8138 (numérique)

Découvrir la revue

Citer ce compte rendu

Déom, E. (1986). Compte rendu de [Reed C. RICHARDSON : Collective Bargaining by Objectives : A Positive Approach. 2nd ed., New Jersey, Prentice-Hall, 1985, 325 pp., ISBN 0-13-140476-8-01]. Relations industrielles / Industrial Relations, 41(1), 191-192. https://doi.org/10.7202/050191ar

Tous droits réservés (C) Département des relations industrielles de l'Université Laval, 1986
Ce document est protégé par la loi sur le droit d'auteur. L’utilisation des services d'Érudit (y compris la reproduction) est assujettie à sa politique d'utilisation que vous pouvez consulter en ligne.

https://apropos.erudit.org/fr/usagers/politique-dutilisation/ 
Le titre II, sur «La carrière professionnelle du salarié», est particulièrement actuel, axé autour de la formation professionnelle et de la gestion (juridique) des carrières, le titre III étant consacré aux «Mouvements de personnel»: mutations au sein d'une même entreprise ou à l'intérieur d'un groupe de sociétés - marché interne de plus en plus sollicité actuellement, ce qui limite les licenciements —, le détachement et le transfert du salarié, la démission, le licenciement (cause réelle et sérieuse, procédure, préavis), la rupture du contrat pour force majeure, et l'apurement de la situation au départ du salarié.

Truffé de conseils pratiques de la plus grande utilité, ce tome premier devait être suivi d'un tome second examinant les relations de travail (discipline, temps, exécution, rémunération du travail, modifications dans la structure juridique de l'entreprise, le licenciement économique) et les relations sociales (institutions représentatives du personnel et syndicats dans l'entreprise, participation, revendications et conflits).

L'importance des thèmes traités et la démarche empruntée font de ce second tome un écrit aussi attendu que le fut le premier. C'est alors que nous disposerons d'une vue globale de l'édifice. Mais la seule lecture du tome I me fait penser que le pari engagé par Michel Despax et Jean Pélissier est en voie d'être gagné. Les gestionnaires et relationnistes, dont je suis, l'ont déjà confirmé. Quant aux juristes eux-mêmes, ceux qui proclament, curieusement, la mort du droit du travail, trouveront-ils, ici, des raisons d'espérer?

\section{Dimitri WEISS}

Université de Paris I Panthéon-Sorbonne

Collective Bargaining by Objectives: A Positive Approach, par Reed C. Richardson, deuxième édition, Prentice-Hall, New-Jersey, 1985, 326 pp., ISBN 0-13-140476-8 01

Cette deuxième édition se veut construite autour d'une application des principes de la gestion par objectifs au processus de la négociation collective. Le volume se divise en quatre parties. La première présente quelques données historiques et juridiques sur la négociation collective aux Etats-Unis (chapitres 1 à 5). Ainsi, après avoir effectué une synthèse des différents événements, de 1790 à aujourd'hui, qui ont contribué à façonner le portrait actuel de la négociation collective (chap. 1 et 2), l'auteur présente une vue globale de l'environnement légal de la négociation aux Etats-Unis (chap. 3). On y retrouve ainsi, sous forme de tableaux, quelques dispositions de deux législations nationales, le National Labor Relations Act et le Civil Service Reform Act, et de quatre législations des états de Hawaï, du Minnesota, de New-York et de l'Iowa, applicables au secteur public. Puis, l'auteur présente les parties à la négociation collective (chap. 4) et enfin, la convention collective (nature, portée et surtout contenu (chap. 5).

La deuxième partie du volume traite du processus de la négociation collective (chapitres 6 à 15). Richardson présente d'abord, très brièvement, son concept de négociation collective par objectifs (chap. 6). Il précise par la même occasion que le succès de la négociation dépend essentiellement de deux facteurs: d'abord, la qualité de la phase préparatoire puis des habiletés des négociateurs. Le chapitre 7 vient ensuite préciser la méthode suggérée par l'auteur, étape par étape. L'objectif de la phase préparatoire est ainsi de réunir toutes les informations nécessaires pour conduire efficacement les négociations (informations tirées des négociations précédentes, des comparaisons avec d'autres conventions, des caractéristiques des employés de l'unité, de l'application de la convention). Les deux chapitres suivants consistent essentiellement en des exemples de tableaux permettant la cueillette et l'analyse de toutes ces informations. Le chapitre 10 précise, par rapport aux différentes législations en traitant, les clauses de 
conventions obligatoires, volontaires et prohibées. L'identification des sujets de négociation constitue ainsi le premier pas pour développer le plan détaillé des négociations. Les prochaines étapes de la méthode consistent à assigner des priorités à chaque sujet (chap. 11) à ranger ces sujets ou objectifs de négociations selon une hypothèse de règlement optimiste, réaliste ou pessimiste (chap. 12), à préciser les positions initiales de négociation (chap. 13) et enfin, d̀ effectuer une vérification des informations (chap. 14). Le dernier chapitre de cette partie traite, en quelques pages, de la négociation comme telle.

La troisième partie du volume aborde l'administration de la convention collective (chapitres 16 à 18). L'auteur situe d'abord le grief selon les législations déjà citées (chap. 16) puis s'attarde à donner des exemples de situations pouvant donner lieu à un grief. Le chapitre suivant traite de la procédure interne (étapes, définition du grief, parties intéressées) et encore là, l'auteur fournit des exemples de différentes procédures. Enfin, l'arbitrage des griefs est abordé selon la même méthode que dans les précédents chapitres, c'est-à-dire avec beaucoup d'exemples tirés de différentes conventions collecitves.

La quatrième et dernière partie (chapitre 19) passe en revue les différents moyens possibles pour résoudre les conflits tant lors de la négociation que lors de l'application de la convention collective. Un exercice de négociation collective complète le volume.

Le volume de Richardson ne peut prétendre à une explication théorique de la négociation. Il fournit essentiellement des données techniques et des instruments utiles pour les personnes appelées à mener des négociations. Il aurait ainsi été préférable que l'auteur présente son volume comme étant un outil de négociation, ce qu'il est effectivement, plutôt que d'essayer d'enrober le tout sous un nouveau concept, nulle part défini par ailleurs, de négociation collective par objectifs. Les tableaux et grilles suggérés pour cueillir et analyser l'information utile à la négociation témoignent d'une approche pratique, un peu comptable, de la négociation.

Il est ainsi un peu dommage que l'auteur se soit senti obligé d'enrober son expérience de références théoriques pas toujours «actuelles» (il cite particulièrement les Webb). Malgré un parti pris évident pour la négociation collective, il demeure quand même surprenant que, dans cette seconde édition de 1985, l'auteur ne souligne nulle part les conséquences de la diminution du taux de syndicalisation, particulièrement aux Etats-Unis, sur la négociation collective. Il prend ainsi fermement position, à contre-courant des travaux de Kochan et als du MIT, sur le rôle prépondérant de la négociation collective dans les relations entre les parties. «Less attention is being paid today to alternatives and substitutes for collective bargaining and more to innovative methods of improving the effectiveness of collective bargaining" (p. 28).

En résumé, il s'agit d'un volume écrit par un praticien qui s'adresse à d'autres praticiens et, à ce titre, il pourra effectivement s'avérer utile surtout aux praticiens américains, compte tenu des références à la législation américaine, et aussi, mais dans une moindre mesure, à des négociateurs impliqués dans d'autres régimes de négociation collective.

Esther DÉOM

Université Laval 